Relations industrielles

Industrial Relations

\title{
The Trade Union Movement of Canada 1827-1959, par Charles Lipton, Canadian Social Publications Limited, Montreal, 1966, 366 pages.
}

\section{Jean-Réal Cardin}

Volume 24, numéro 1, 1969

URI : https://id.erudit.org/iderudit/027998ar

DOI : https://doi.org/10.7202/027998ar

Aller au sommaire du numéro

Éditeur(s)

Département des relations industrielles de l'Université Laval

ISSN

0034-379X (imprimé)

1703-8138 (numérique)

Découvrir la revue

Citer ce compte rendu

Cardin, J.-R. (1969). Compte rendu de [The Trade Union Movement of Canada 1827-1959, par Charles Lipton, Canadian Social Publications Limited, Montreal, 1966, 366 pages.] Relations industrielles / Industrial Relations, 24(1), 218-219. https://doi.org/10.7202/027998ar

Tous droits réservés (c) Département des relations industrielles de l'Université Laval, 1969
Ce document est protégé par la loi sur le droit d'auteur. L'utilisation des services d'Érudit (y compris la reproduction) est assujettie à sa politique d'utilisation que vous pouvez consulter en ligne.

https://apropos.erudit.org/fr/usagers/politique-dutilisation/ 


\section{RECENSIONS}

L'homme en situation industrielle, par Philippe Muller et Paul Silberer, Payot, Paris, 1968, 475 pages.

Muller et Silberer nous font part d'une foule de recherches qu'ils ont effectuées au cours des dernières années, sur l'homme confronté aux multiples techniques industrielles. Non seulement rapportent-ils de nombreuses techniques mais ils apportent d'intéressantes distinctions entre certains concepts qui portent à ambiguité. De plus, ils proposent d'excellentes références sur les sujets qu'ils discutent.

Les principaux sujets de leur étude sont les suivants: la psychologie industrielle, les besoins sociaux, l'entreprise, l'environnement des tâches, l'accès au travail, l'intégration du travailleur à l'entreprise, la notation du personnel, les motivations, les conflits, la participation et l'interaction des individus.

Ce volume se veut d'abord un manuel de psychologie où l'on étudie les répercussions des techniques de relations industrielles sur les individus. Or c'est l'une des rares publications françaises dans son genre et certainement la plus complète. En d'autres termes, c'est une publication que tous devraient avoir dans leur bibliothèque, puisqu'elle est d'une grande valeur.

\section{Pierre BRIEN}

Dictionnaire de l'économie contemporaine, par Fernand Baudhuin. Collection Economie moderne, Editions Marabout, Verviers, 1968, 302 pages (Distribué au Canada par D. Kasan, 226 est Christophe Colomb, Québec).

Personne ne peut de nos jours se passer d'un minimum de connaissances économiques. Tous en ont besoin soit dans leur activité professionnelle, soit pour comprendre ce qui se passe dans le monde

L'économique, comme les autres sciences, a son langage particulier qui souvent n'est accessible qu'aux inities.
Le Dictionnaire de l'économie contemporaine ne cherche pas à être un ouvrage réservé aux spécialistes ou destiné à faire avancer la science. Mais il réussit à être un instrument d'information à l'usage du grand public. On y trouve donc non seulement la définition des principaux termes en usage, mais encore des explications et des renseignements. On y trouve aussi des brèves notices sur les économistes qui ont contribué et qui contribuent encore au développement de la science économique. L'auteur a mis l'accent sur l'économie contemporaine en y introduisant ce qui regarde les méthodes nouvelles en usage ainsi que les institutions économiques et financières.

A l'encontre de ce que nous fournissent habituellement les ouvrages européens de langue française, et probablement parce que son auteur est belge, le IDictionnaire de l'économie contemporaine possède un caractère universel et est ouvert à ce qui se passe en dehors de l'Europe continentale. Pour nous du Canada, c'est un mérite appréciable.

Ce dictionnaire, enfin, présente dans un appendice la liste des institutions membres de l'Association internationale des sciences économiques, celle des entreprises d'intérêt mondial, les grandes inventions d'intérêt industriel et les grandes dates d'intérêt économique.

Le Dictionnaire de l'économie contemporaine ne remplace pas les encyclopédies, mais il a l'avantage de mettre à la portée de tous pour un prix très accessible un instrument de travail précis, utile et de consultation rapide.

\section{Gérard DION}

The Trade Union Movement of Canada, 1827-1959, par Charles Lipton, Canadian Social Publications Limited, Montreal, 1966, 366 pages.

Les ouvrages qui portent sur l'histoire du syndicalisme dans son cheminement global sont relativement rares au Cana- 
da et plus particulièrement au Québec. $\mathrm{Ne}$ serait-ce que pour cette seule raison, le livre de $\mathbf{M}$. Lipton représente une contribution valable à la littérature ouvrière en notre pays.

Il faut rappeler que la plupart des études générales de l'histoire du mouvement ouvrier au Canada, celles parmi les plus utilisées, s'arrêtent aux années de l'entre-deux-guerres ou à celles qui l'ont immédiatement suivie; on pense, par exemple aux travaux de Margaret Mackintosh et de Harold Logan, entre autres.

Enfin, les quelques ouvrages écrits en français et portant sur l'ensemble de l'histoire syndicale au Canada ne sont pas, dans une bonne mesure, le résultat de recherches de première main et sont plutôt schématiques.

Cet ouvrage fait montre d'une certaine recherche des documents pertinents, officiels et publics, documents émanant des organisations ouvrières elles-mêmes, ainsi que journaux de l'époque.

Il s'agit évidemment d'un ouvrage qui prend le contrepied des versions officielles de certaines grandes centrales canadiennes et de l'opinion prévalente dans les grands organes d'information, particulièrement en ce qui a trait aux relations syndicales canado-américaines, aux luttes entre la "gauche" et la "droite" au sein de notre mouvement ouvrier, etc...

L'auteur apporte une vue personnelle et engagée, qui faisait peut-être défaut dans ce genre d'entreprises au Canada.

Il ne s'agit pas d'une oeuvre de caractère académique mais bien plutôt de l'interprétation d'un homme d'action, ayant déjà été engagé lui-même dans le le mouvement ouvrier.

La principale faiblesse de ce travail, c'est le traitement plus que sommaire accordé aux événements propres au Québec, au syndicalisme national catholique qui deviendra la Confédération des Syndicats Nationaux (CSN) en 1960, ainsi qu'à l'étude de ce milieu particulier qui ne peut être escamoté dans une histoire générale du mouvement ouvrier au $\mathrm{Ca}$ nada.

L'auteur consacre trois (3) pages à la C.T.C.C. dans son chapitre 12 , et se limite dans son dernier chapitre à cer- tains événements, tels certaines grèves, certes parmi les plus importants, mais qui sont insuffisants pour constituer un traité historique valable sur le sujet. Ce n'est pourtant pas que les sources manquent sur une telle matière.

Si on tient compte de ces déficiences, il reste, comme nous le soulignons au début, que l'ouvrage de M. Lipton apporte une contribution qu'il nous faut souligner à l'examen d'une institution sur laquelle encore beaucoup reste à explorer et à interpréter.

\section{Jean-Réal CARDIN}

La C.G.T. par A. Barjonet, Seuil, Paris, 1968, 176 pages.

La CGT a connu des débuts difficiles à cause du rassemblement d'unités hétérogènes qui est à son origine: (1895).

L'année 1936, dite de l'unité retrouvée, rappelle de précieuses victoires pour le syndicalisme français: notamment l'obtention de la semaine de 40 heures et les deux semaines de vacances payées. Le contexte d'après crise favorisait ce règlement.

La guerre de $1939-45$ soulèvera le problème communiste et l'après-guerre, surtout 1947, connaîtra l'aide américaine aux syndicats non-communistes.

La CGT, avec ses 2 millions de membres n'en demeure pas moins l'organisation syndicale la plus influente et la plus significative du monde ouvrier français.

C'est en 1955 à Strasbourg que Marcel David fondait le premier "Institut du Travail".

L'auteur nous fait sentir le besoin d'unité syndicale en France. La CGT comme plusieurs autres syndicats sont conscients de l'absolue nécessité de l'unité syndicale pour obtenir satisfaction sur des revendications communes. Cependant ce que tous craignent dans l'unité, c'est d'être éliminé ou réduit à l'impuissance.

Enfin, il souligne les relations de la CGT avec les événements de mai.

Pierre BRIEN 